\title{
Compartmental Model Region
}

National Cancer Institute

\section{Source}

National Cancer Institute. Compartmental Model Region. NCI Thesaurus. Code C94873.

A distinct region or state for a particular chemical substance at a cellular level taken to have uniformity throughout but differing from other regions or states. 